\title{
Relación entre la complejidad técnico-táctica en la sesión de entrenamiento y la carga interna en baloncesto femenino
}

\section{Relationship between technical-tactical complexity in the training session and internal load in female basketball.}

\author{
Vallés Ortega, C. ${ }^{1}$, Fernández-Ozcorta, E.J. ${ }^{2}$ y Fierro Suero, S. ${ }^{3}$ \\ 1 Centro de Técnicos Deportivos Alándalus. \\ 2 Escuela Deporte (Grupo DOGESPORT) y miembro del grupo de investigación E-MOTION (HUM-643). \\ 3 Beca Colaboración master oficiales. Master en investigación en educación física y ciencias del deporte
}

\begin{abstract}
Resumen: El objetivo de este trabajo fue analizar la Escala de Valoración de la Carga del entrenamiento Técnico-Táctico (Coque, 2009) y su relación con parámetros de carga interna. En la actualidad, se plantea un modelo de planificación que se caracteriza por la preocupación de mejorar la interpretación que el sujeto hace de lo que sucede a su alrededor durante la competición (Martín Acero, Seirul-lo Vargas, Lago Peñas, y Novoa Lalín, 2013), para así modificar la respuesta y a su vez, el esquema motriz. El estudio contó con las jugadoras del equipo de Liga Femenina del Club Baloncesto Conquero, compuesto por 12 jugadoras, con edades comprendidas entre 17 y 33 ańos $(\mathrm{M}=21.91 ; \mathrm{SD}=4.81)$. Los datos fueron recogidos durante los dos últimos meses de competición que se corresponden con los meses de febrero y marzo de 2016. Para obtener los datos necesarios se utilizaron la escala de valoración subjetiva de la carga de entrenamiento Técnico-Táctico (Coque, 2009), la percepción subjetiva del esfuerzo (Borg, 1970; 1998; Borg \& Kaijser,2006) y la calidad de la recuperación (Kentta \& Hassmen, 1998). Los resultados mostraron que sólo la densidad del entrenamiento es el único marcador que podría explicar, de forma importante (91.4\%), el esfuerzo percibido de las jugadoras. Según los datos obtenidos la percepción subjetiva del esfuerzo podría ser utilizada para la monitorización del entrenamiento en cuanto a la complejidad técnico-táctica del mismo.

Palabras claves: carga de entrenamiento Técnico-Táctico; percepción subjetiva del esfuerzo; calidad de la recuperación; baloncesto femenino.
\end{abstract}

Abstract: The objective of this work was to analyze the rating scale loadTactical Technical (Coque, 2009) Training and Its Relationship with internal load parameters. Currently, a planning model characterized by concern to improve the interpretation that the subject does what happens around them during the competition (Martin Steel, Seirul-lo Vargas, Lake Peñas, and Novoa Lalin arises, 2013), to modify the response and in turn, the drive scheme. The study involved the players formed Women's League team Basketball Club Conquero, composed of 12 players, aged between 17 and 33 years $(M=21.91, S D=4.81)$. Data were collected during the last two months of competition in February and March. Par obtain the data necessary the following scales of subjective assessment of the burden of technical and tactical training (Coque, 2009), perceived exertion (Borg, 1970; 1998; Borg \& Kaijser,2006) and quality of recovery (Kentta \& Hassmen, 1998) were used. The results showed that only the density of training is the only marker that could explain significantly $(91.4 \%)$ perceived effort of the players. According to data from the perceived exertion, it could be used for monitoring training regarding technical and tactical complexity.

Keywords: technical and tactical load training; perceived exertion; recovery quality; female basketball.

\section{Introducción}

El entrenamiento es entendido como el proceso en el que el sujeto es sometido a estímulos (carga) conocidos y planificados que provocan en el sujeto una fatiga controlada que, tras los suficientes y adecuados procesos de recuperación, conllevan una mejora del rendimiento específico para cada disciplina deportiva (Borresen \& Lambert, 2009).

Para entender más a fondo la carga de entrenamiento, tendríamos que analizar el concepto de fatiga, y los medios utilizados para controlar su aparición. Asimismo su definición se torna confusa, debido a la complejidad de los mecanismo que la producen y a las múltiples causas que provocan su aparición (Shen, Barbera, \& Shapiro, 2006). Conde-González

Dirección para correspondencia [Correspodence address]: Carlos Vallés Ortega. Basilio Marquínez, no 1, 21007, Huelva (Espańa). E-mail: carlos. valles@centrosalandalus.com
(2012) entiende la fatiga como mecanismo de seguridad encaminado a la prevención de lesiones, acentuando la consideración de fatiga como un estado funcional de significación protectora, transitoria y reversible, expresión de una respuesta de índole homeostática, a través de la cual se impone de manera ineludible la necesidad de cesar o, cuando menos, reducir la magnitud del esfuerzo o la potencia del trabajo que se está efectuando (Barbany, 2002). Siendo el control y monitoriazación de dicha fatiga, el medio para prevenir el sobreentrenamiento. Es por esto que, diferentes autores (e.g., Abbiss y Laursen, 2005; Conde-González, 2012) abordan el análisis de la fatiga desde diferentes orientaciones: biomecánica, fisiológica y psicológica.

Según la orientación biomédica, la fatiga es considerada como un déficit de fuerza producidas por un músculo que afecta a patrones de movimientos básicos de cada modalidad 
deportiva y, por lo tanto, determina la ejecución técnica de los gestos deportivos de cada modalidad (Conde-González, 2012). Por otra parte, encontramos la orientación fisiológica. Esta es definida como el fallo del organismo el cual se ve reflejado en una disminución del rendimiento que a su vez es producido por un exceso de consumo de energía, al igual que la depleción de hormonas, neurotransmisores o substratos esenciales para la función fisiológica (Aaronson, Teel, \& Cassmeyer, 1999; Conde-González, 2012). A su vez, otros autores como López-Chicharro y Fernández (2006), la definen como la disminución de la capacidad para generar fuerza máxima y potencia máxima, independientemente de que la intensidad del esfuerzo pueda, o no ser, mantenida.

Dentro de la especificidad deportiva, a la hora de la monitorización del entrenamiento, se hace tradicionalmente a través de la carga externa y la carga interna (Malone et al., 2015). La carga externa se entiende como la medida objetiva que realiza el sujeto en un entrenamiento o competición (Mujika, 2013). Los datos que engloba esta carga externa lo componen indicadores como: la tarea, la duración, número de sesiones, velocidad de ejecución, etc. (Harre y Barsch 1982). Mientras, la carga interna es referida a la respuesta fisiológica que tiene el sujeto como consecuencia del estímulo que supone la carga externa (Schelling, 2012). Estas pueden encontrarse como marcadores fisiológicos, tales como: la frecuencia cardiaca máxima (Fc Max), niveles de lactato, consumo máximo de oxígeno $\left(\mathrm{VO}_{2} \mathrm{Max}\right)$, etc.

De forma general, el estudio de estas variables ha sido muy prolífero en deportes como el baloncesto masculino dado que el estudio de la carga de entrenamiento no siempre requiere de grandes esfuerzos económicos y medios técnicos para su monitorización. Sin embargo, existe un vacío sobre su homólogo femenino.

En la literatura es frecuente encontrar estudios que emplean instrumentos como cuestionarios o escalas, que precisan la cuantificación subjetiva sobre diferentes variables. En este sentido, han proliferado dichos instrumentos que ayudan a recolectar datos de carga percibida por el jugador. Entre los más empleados encontramos Borg CR10 Scale versión modificada de la Rate of Percived Exertion (RPE) (Borg, 1970; 1998; Borg \& Kaijser,2006). Este sencillo método se ha desarrollado para cuantificar las cargas de entrenamiento de una variedad de diferentes modalidades de entrenamiento en un número arbitrario simple (Foster, Daines, Hector, Snyder, \& Welsh, 1996).

Uno de los objetivos de la monitorización es saber cuál es el momento adecuado de aplicar otra carga de entrenamiento. Es lógico pensar que para aumentar el rendimiento se pueden lograr con un aumento de las cargas de entrenamiento. Estos aumentos sólo se pueden tolerar a través de períodos intercalados de descanso y recuperación periodización de entrenamiento (Bishop, Jones, \& Woods, 2008). Para ello existe un método práctico y útil que se usa para medir el proceso de recuperación, nos referimos a la Escala de la Calidad de la Recuperación Total (TQR) de Kentta y Hassmén (1998). Esta escala usa la misma escala que la escala RPE (Borg \& Kaijser, 2006; Borg, 1970; 1998) que proporciona un medio para medir la recuperación psicofisiológica.

Hasta la fecha las investigaciones han aportado evidencias científicas que han permitido establecer pautas de recuperación ante cargas que inciden en las diferentes cualidades condicionales, sim embargo no sucede igual con las cargas mentales ya sean cognitivas o emocionales (Cárdenas, Perales, \& Alarcón, 2014). Según Conde-González (2012) la fatiga mental es el déficit de subjetiva de energía física o psíquica, percibida por el individuo, que interfiere en sus actividades usuales y deseadas. Siguiendo en esta líneas autores como Vélez y Conde-González (2015) definen la fatiga mental como el sumatorio del esfuerzo mental a lo largo del tiempo, y su aparición y manifestaciones muestran cierto paralelismo con las de la fatiga fisiológica. Según Conde-González (2012) esto significa que la fatiga se entiende como una experiencia subjetiva, basada en el autocontrol del deportista, y que depende de la precepción del agotamiento físico, mental o ambos (Bol, Duits, Hupperts, \& Vlaeyen, 2009). Conde-González (2012) relata que la sensación de esfuerzo refleja, más o menos la calidad del impulso motor desde el córtex cerebral a la motoneurona de la médula espinal.

Después de tratar la fatiga desde un aspecto psicológico definiremos el contenido de carga mental, completando así el concepto de carga tratado anteriormente como carga interna y externa. Cárdenas et al., (2014) la define como el costo de recursos para un individuo dadas sus capacidades, mientras consigue un nivel de rendimiento determinado en una tarea con demandas específicas, constando esta de dos componentes cognitivos y emocionales. Siguiendo en esta misma línea Conde-González (2012) la entiende como el resultado de la carga a la suma la carga cognitiva, correspondiente al esfuerzo cognitivo desarrollado por parte del ejecutivo central, y la carga emocional, entendida como el efecto emocional derivado de dicho esfuerzo, así como la retroalimentación resultante de la realización de la tarea cognitiva.

El baloncesto es una actividad deportiva colectiva que se desarrolla en un entorno inestable, con modificaciones constante de algunos de sus elementos, lo que genera un elevado grado de incertidumbre para sus jugadores (Lorenzo, Ibáñez, \& Ortega, 2009), este modalidad deportiva exige al jugador evaluar el contexto, decidir el plan de acción y ejecutarlo (Lorenzo et al., 2009), por lo tanto supone fundamental percibir la situación de juego, evitando procesar la información irrelevante para la toma de decisiones.

Por todo lo anterior mencionado supone fundamental encontrar escalas, que nos ayuden a controlar las carga de entrenamiento a nivel técnico táctico. Estas escalas recogen 
datos sobre la carga que supone el entrenamiento a nivel psicológico y fisiológico, o como se ha argumentado, la carga interna. Pero uno de los hándicaps de estas escalas es la imposibilidad de recoger aspectos técnico-tácticos que, al fin y al cabo, suponen la carga externa a la que están sometidos los deportistas. En relación a esta preocupación, Coque (2009) propuso una escala de valoración de las cargas de entrenamiento mediante el análisis técnico-táctico. Este instrumento permite registrar y cuantificar la carga del entrenamiento desde esta nueva perspectiva y no para la cuantificación de los entrenamientos físicos (García, Parejo, \& Cañadas, 2010).

Tras los estudios revisados, el objetivo del estudio fue analizar la Escala de Valoración de la Carga del entrenamiento Técnico-Táctico (Coque, 2009) y su relación con parámetros de carga interna, a través de las escalas Borg CR10 y TQR. Se teoriza que el instrumento citado puede ser una herramienta válida para estimar la carga interna y la calidad de la recuperación de los deportistas.

\section{Material y método}

\section{Participantes}

La muestra del estudio estuvo formada por el equipo de Liga Femenina del Club Baloncesto Conquero, compuesto por 12 jugadoras, con edades comprendidas entre 17 y 33 años $(\mathrm{M}=21.91 ; \mathrm{SD}=4.81)$. Los datos fueron recogidos durante de los dos últimos meses de competición de febrero y marzo.

\section{Instrumentos}

Valoración subjetiva de la carga de entrenamiento TécnicoTáctico (Coque, 2009). Este instrumento pretende valorar las cargas de entrenamiento a través de los siguientes apartados: 1) Grado de oposición donde el índice superior está en la igualdad numérica llegando hasta el mínimo valor en las situaciones sin ninguna oposición. 2) Densidad de la tarea. Éste hace referencia a conocer la relación existente entre el tiempo de trabajo y el tiempo de recuperación. 3) Número de ejecutantes implicados simultáneamente. Se valora como el propio ejercicio induce a la participación de un número de jugadores concreto. 4) Carga competitiva (emotiva, psicológica). se tiene en cuenta el nivel de competitivo de las tareas desarrolladas. 5) Espacio y dimensiones en el que se desarrolla el ejercicio. Pretende valorar la cantidad de espacio dedicado a la tarea. 6) Implicación cognitiva (carga táctica). Valora que el jugador además de realizar las acciones habituales de juego, tenga que realizar un esfuerzo extra en movimientos específicos (defensivos u ofensivos) en los que la coordinación colectiva sea importante. Estas seis dimensiones se valoran de 0 a 4 puntos. Donde 0 representa la valoración mínima de la dimensión y 4 la máxima (e.g., Carga competitiva de un 1 contra 0 tendría una valoración de 0 ; sin embargo, un partido $5 \times 5$ se le aplicaría una valoración de 4). Cada tarea se valora a través de las diferentes dimensiones sumando el total de puntos que la tarea obtiene de cada una. A su vez, la suma de todas las tareas cuantificadas, aporta la carga de entrenamiento total. Para la valoración total del entrenamiento, se tomó la cuantificación de la escala, con el tiempo real de trabajo en el ejercicio, es decir, se multiplica el total de puntos por el tiempo real). Con ello se obtiene el valor de cada ejercicio.

Borg CR10 Scale versión modificada de la Rate of Percived Exertion (RPE) (Borg \& Kaijser, 2006; Borg, 1970; 1998). Dicho instrumento cuantifica la carga de entrenamiento a través de una escala que va desde los valores 1 a 10 puntos catalogados desde "muy muy ligero" hasta "muy muy duro.

Total Quality Recovery (TQR) de Kentta \& Hassmen (1998). Es una escala que permite que el jugador valore su recuparación tras su último entrenamiento o partido. La escala puntúa entre 6-20, en donde 20 representa el máximo nivel de recuperación y el 6 , una mínima recuperación.

\section{Procedimiento}

Antes de pasar las escalas se le solicitó al club la pertinencia del estudio, además de pedir el consentimiento informado a aquellos padres de las jugadoras menores de edad. En la recolección de los datos siempre estuvo presente algunos de los investigadores del estudio. La toma de datos se detalla a continuación. Durante las sesiones se cuantificaba cada tarea de entrenamiento y el sumatorio total de ellas a partir de la escala Subjetiva de la Carga de Entrenamiento Técnico-Táctico. Tras la conclusión del entrenamiento, 20 minutos después, se les suministraba la RPE respecto al entrenamiento realizado. La recolección también se realizaba el día posterior a la sesión de entrenamiento en la cual se les preguntaba por la recuperación a través de la escala TQR.

\section{Análisis estadístico}

El análisis estadístico realizado estuvo dividido en dos pasos. En un primer paso se analizaron los estadísticos descriptivos y de correlaciones. En un segundo momento, se utilizó la regresión lineal (paso a paso) para conocer qué variables y cuánto predecía el esfuerzo subjetivo percibido y la calidad de la recuperación.

Los datos fueron analizados a través del paquete estadístico SPSS 20.0, empleando un nivel significación asumido de $p<.05$.

\section{Resultados}

En la tabla 1 se muestra los estadísticos descriptivos de las variables de estudio y los resultados del análisis de correlación. 
El estudio de correlación muestra que la Escala de Valoración de la Carga del entrenamiento Técnico-Táctico presenta correlación muy fuerte (entre .91 y .96) con la RPE, salvo la dimensión del grado de oposición de la tarea. También se puede indicar que no existen correlaciones entre la escala TQR y la escala de Valoración de la Carga del entrenamiento Técnico-Táctico.

Tabla 1. Análisis de correlaciones entre las variables de estudiadas de carga externa e interna.

\begin{tabular}{|c|c|c|c|c|c|c|c|c|c|c|c|c|}
\hline & Variables & M & DT & 1 & 2 & 3 & 4 & 5 & 6 & 7 & 8 & 9 \\
\hline 1 & Oposición & 5.6 & 8.17 & - & $.895^{*}$ & $.886^{*}$ & $.923^{*}$ & & & $.892^{*}$ & & \\
\hline 2 & Densidad & 8.6 & 6.47 & & - & $.996^{\dagger}$ & $.893^{*}$ & $.953^{*}$ & $.971^{\dagger}$ & $.999^{\dagger}$ & $.963^{\dagger}$ & \\
\hline 3 & Ejecutantes & 8.6 & 6.88 & & & - & & $.957^{*}$ & $.979^{\dagger}$ & $.999^{\dagger}$ & $.941^{*}$ & \\
\hline 4 & Competitiva & 2.0 & 2.12 & & & & - & & & $.879^{*}$ & $.908^{*}$ & \\
\hline 5 & Cognitiva & 11.6 & 6.58 & & & & & - & $.993^{\dagger}$ & $.956^{*}$ & $.939^{*}$ & \\
\hline 6 & Espacios & 14.0 & 9.80 & & & & & & - & $.975^{\dagger}$ & $.927^{*}$ & \\
\hline 7 & Total & 50.2 & 38.1 & & & & & & & - & $.956^{*}$ & \\
\hline 8 & RPE & 3.12 & .54 & & & & & & & & - & \\
\hline 9 & TQR & 14.88 & .57 & & & & & & & & & - \\
\hline
\end{tabular}

Notas: $\mathrm{M}=$ media; DT= desviación típica; RPE= Escala de esfuerzo percibido; TQR=Total quality recovery; $\nmid p<.01 ;{ }^{*} p<.05$

En un segundo paso, con la aplicación de la regresión lineal, los resultados mostraron que, independientemente de existir correlación, solo la densidad del entrenamiento explica el 91.4\% $(\mathrm{F}(1.161)=38.67$; $\mathrm{p} \leq 0.05)$, del esfuerzo percibido. Según dichos datos con sólo conocer la densidad podríamos tener una estimación muy próxima del esfuerzo percibido por las jugadoras. Sin embargo, las demás dimensiones no nos aportarían datos suficientes para poder estimar el esfuerzo que perciben las jugadoras.

\section{Discusión}

El objetivo principal del estudio fue analizar la complejidad táctica y su relación con parámetros de carga interna. Asimismo, se teorizó que la Escala de Valoración de la Carga del entrenamiento Técnico-Táctico (Coque, 2009) pudiese ser una herramienta válida para estimar la carga interna. En este sentido, la hipótesis inicial no se cumplió. Sólo la densidad del entrenamiento logró explicar la carga interna estimada con los instrumentos seleccionados.

En los últimos años la toma de decisiones y el análisis de los procesos cognitivos en el deporte ha sido una preocupación creciente y base de diferentes modelos de planificación. Ejemplo de ello es el modelo de (Arjol, 2012; Martín Acero, Seirul-lo Vargas, Lago Peńas, \& Novoa Lalín, 2013) basados en que las personas conocen la realidad a través de modelos que construyen para poder explicarla y a su vez, estos modelos son susceptibles de ser mejorados y cambiados. Cárdenas et al. (2014) van más allá y sugieren que la carga mental debe ser planificada cuidadosamente, ya que el fracaso de las adaptaciones en este campo puede llevar a resultados no deseados como sobreentrenamiento o burnout. La dificultad de registrar los aspectos técnico-tácticos ha relegado este ámbito a un segundo plano respecto a variables físicas y psicológicas. En relación a esta preocupación y debido a la falta de instrumentos de cuantificación de la carga técnico-táctica en baloncesto, (Coque, 2009) propuso la escala de valoración de las cargas de entrenamiento técnico-táctico como un instrumento que tratara de objetividad y cuantificar la carga del entrenamiento desde esta nueva perspectiva y no para la cuantificación de los entrenamientos físicos (García et al., 2010). Hasta ahora, la monitorización de las cargas de entrenamiento se ha centrado en tratar de cuantificar las cargas externas e internas, como por ejemplo son el caso de las escalas subjetivas como la escala RPE (Borg, 1970; 1998; Borg \& Kaijser,2006) o TQR (Kentta \& Hassmen, 1998) que se han relacionado con la monitorización de los parámetros físicos (e.g., Aoki et al., 2016; Brink, Nederhof, Visscher, Schmikli, \& Lemmink, 2010; Fanchini, Ghielmetti, Coutts, Schena, \& Impellizzeri, 2015; Kentta \& Hassmen, 1998; Thorpe et al., 2016).

Al tratarse la escala de valoración de las cargas de entrenamiento técnico-tácticas de un instrumento relativamente novedoso, no se han encontrado estudios que relacionen si esta herramienta tiene su correspondiente relación con la carga física del entrenamiento en baloncesto, por tanto, el objetivo principal de este estudio fue analizar la relación entre la complejidad táctica, medida mediante la Escala de Valoración subjetiva de la Carga del entrenamiento Ténico-Táctico (Coque, 2009); y la carga interna para que la se utilizó la RPE (Borg, 1970; 1998; Borg \& Kaijser,2006) y la escala TQR (Kentta \& Hassmen, 1998).

Los resultados del trabajo presentado han desvelado que 
la Escala de Valoración de la Carga del entrenamiento Técnico-Táctico presenta una correlación muy fuerte (entre .908 y .963) con la RPE, pero no con la TQR. Ya que estudios previos (Aoki et al., 2016; Brink et al., 2010) han mostrado la relación entre la RPE, la TQR y su valor predictivo como indicadores de la carga de entrenamiento, estos hallazgos parecen apuntar que el modelo de cuantificación técnico-táctico (Coque, 2009) puede ser una buena herramienta que guarde una relación importante entre la carga técnico-táctica y la carga física de las sesiones de entrenamiento. En este sentido, se recomienda seguir investigando sobre la relación entre los instrumentos planteados con diferentes muestras para tratar de seguir avanzando hacia la creación y utilización de instrumentos globales de monitorización del entrenamiento deportivo.

A modo de conclusión, los datos muestran que la Escala de Valoración de la Carga del entrenamiento Técnico-Táctico (Coque, 2009), podría ser utilizado como un instrumento de monitorización del entrenamiento de la carga interna en baloncesto femenino, avanzando así hacia instrumentos más globales y asequibles para la mayoría de los clubes de baloncesto. Esta herramienta facilita la implantación necesaria de sistemas de medición que ayuden a evolucionar desde criterios subjetivos, que han dirigido el trabajo de entrenadores y preparadores físico, hasta criterios más rigurosos, objetivos (Zabala, Chirosa, Chirosa, \& Viciana, 2002).

En consecuencia, se plantea la necesidad de estudios sobre este colectivo se hace notable para la mejora del rendimiento y de la salud, es fundamental conocer y comprender cuales son los factores limitantes de la disciplina deportiva que se trate.

\section{Referencias}

1. Aaronson, L., Teel, C., \& Cassmeyer, V. (1999). Defining and measuring fatigue. The Journal of Nursing Scholarship, 31(1), 45-50. Retrieved from http://onlinelibrary.wiley.com/doi/10.1111/j.1547-5069.1999. tb00420.x/abstract

2. Abbiss, C., \& Laursen, P. (2005). Models to explain fatigue during prolonged endurance cycling. Sports Medicine. Retrieved from http://link. springer.com/article/10.2165/00007256-200535100-00004

3. Aoki, M. S., Torres, R. L., Marcelino, P. R., Drago, G., Carling, C., Bradley, P. S., \& Moreira, A. (2016). Monitoring training loads in professional basketball players engaged in a periodized training programme. Journal of Strength and Conditioning research/National Strength \& Conditioning Association., 24(3), 597-603.

4. Arjol, J. L. (2012). La planificación actual del entrenamiento en fútbol.: Análisis comparado del enfoque estructurado y la periodización táctica. Acciónmotriz, (8), 27-37.

5. Barbany, J. R. (2002). Fundamentos de fisiología del ejercicio y del entrenamiento (Barcanova). Barcelona.

6. Bishop, P. A., Jones, E., \& Woods, A. K. (2008). Recovery from training: a brief review: brief review. The Journal of Strength \& Conditioning Research, 22(3), 1015-1024.

7. Bol, Y., Duits, A., Hupperts, R., \& Vlaeyen, J. (2009). The psychology of fatigue in patients with multiple sclerosis: a review. Journal of. Retrieved from http://www.sciencedirect.com/science/article/pii/ S0022399908002201

8. Borg, E., \& Kaijser, L. (2006). A comparison between three rating scales for perceived exertion and two different work tests. Scandinavian Journal of Medicine \& Science in Sports, 16(1), 57-69.

9. Borg, G. (1970). Perceived exertion as an indicator of somatic stress. Scandinavian Journal of Rehabilitation Medicine, 2, 92-98.

10. Borg, G. (1998). Borg's perceived exertion and pain scales. Human kinetics.

11. Borresen, J., \& Lambert, M. I. (2009). The quantification of training load, the training response and the effect on performance. Sports Medicine, 39(9), 779-795.

12. Brink, M. S., Nederhof, E., Visscher, C., Schmikli, S. L., \& Lemmink, K. A. (2010). Monitoring load, recovery, and performance in young elite soccer players. The Journal of Strength \& Conditioning Research, 24(3), 597-603.

13. Cárdenas, D., Perales, J., \& Alarcón, F. (2014). La planificación del entrenamiento para la toma de decisiones en los deportes de equipo.
En L. García González y F. Del Villar (Coords.), El entrenamiento táctico y decisional en el deporte (pp.265-293). Madrid: Editorial Síntesis S.A.

14. Conde-González, J. (2012). La interacción de la carga de trabajo física y mental en la percepción de la fatiga física durante y después de un ejercicio físico hasta el agotamiento (Tesis doctoral). Universidad de Granada, Granada.

15. Coque, I. (2009). Valoración subjetiva de la carga del entrenamiento técnico-táctico: una aplicación práctica (y II). Clinic: Revista Técnica de Baloncesto, 22(82), 43-45.

16. Fanchini, M., Ghielmetti, R., Coutts, A. J., Schena, F., \& Impellizzeri, F. M. (2015). Effect of Training Session Intensity Distribution on Session-RPE in Soccer. International Journal, 10(4), 426-430.

17. Foster, C., Daines, E., Hector, L., Snyder, A. C., \& Welsh, R., (1996). Athletic performance in relation to training load. Wisconsin Medical Journal, 95(6), 370-374.

18. García, J., Parejo, I., \& Cañadas, M. (2010). Valoración de la carga de entrenamiento. Una experiencia real con un equipo de baloncesto de liga EBA. Revista Internacional de Deportes Colectivos, 5, 4-17.

19. Harre, D., \& Barsch, J. (2012). Principles of sports training: introduction to the theory and methods of training. Berlin: Sportverlag,

20. Kentta, G., \& Hassmen, P. (1998). Overtraining and recovery: a conceptual model Surentrainement et recuperation: un modele conceptuel. Sports Medicine, 26(1), 1-16. http://doi.org/10.2165/00007256199826010-00001

21. López-Chicharro, J., \& Fernández, A. (2006). Fisiología del Ejercicio. Barcelona: Médica Panamericana.

22. Lorenzo, A., Ibáñez., S., \& Ortega, E. (2009). La intervención del entrenador de baloncesto: investigación e implicaciones prácticas. Aportaciones teóricas y prácticas para el baloncesto del futuro. Sevilla: Editorial Wanceulen.

23. Malone, J. J., Michele, R. Di, Morgans, R., Burgess, D., Morton, J. P., \& Drust, B. (2015). Seasonal training-load quantification in elite English premier league soccer players. International Journal of Sports Physiology and Performance, 10(4), 489-497.

24. Martín Acero, R., Seirul-lo Vargas, F., Lago Peńas, C., \& Novoa Lalín, C. (2013). Causas objetivas de planificación en DSEQ (II): la microestructura (microciclos). Revista de Entrenamiento Deportivo, 27(Ii).

25. Mujika, I. (2013). The alphabet of sport science research starts with Q. International Journal Sports Physiol Perform, 8(5), 465-466. 
26. Schelling, X. (2012). Exigencia en baloncesto: carga externa e interna. Deportes, Salud Y Entrenamiento, 11, 6-23.

27. Shen, J., Barbera, J., \& Shapiro, C. (2006). Distinguishing sleepiness and fatigue: focus on definition and measurement. Sleep Medicine Reviews. Retrieved from http://www.sciencedirect.com/science/article/ pii/S1087079205000444

28. Thorpe, R. T., Strudwick, A. J., Buchheit, M., Atkinson, G., Drust, B., \& Gregson, W. (2016). The Tracking of Morning Fatigue Status Across
In-Season Training Weeks in Elite Soccer Players. International Journal of Sports Physiology and Performance, 11(7), 947-952.

29. Vélez, D. C., \& Conde-González, J. (2015). El papel de la carga mental en la planificación del entrenamiento deportivo. Revista de Psicología, 24(1), 91-100.

30. Zabala, M., Chirosa, I. J., Chirosa, L., \& Viciana, J. (2002). Tecnología y metodología cientifica aplicada al control y evaluación del rendimiento deportivo. Granada: Repografía Digital Granada. 